\title{
Understanding and Realizing Presence in the Presenccia Project
}

Mel Slater

ICREA-

Universitat

Politècnica de

Catalunya and

University

College London

Antonio Frisoli and Franco

Tecchia

Scuola di Studi

Universitari e di

Perfezionamento

Sant 'Anna

Christoph Guger

Guger

Technologies

OEG

Beau Lotto and Anthony Steed University College London

Gert

Pfurtscheller and Robert Leeb Graz University of Technology

Miriam Reiner Israel Institute of Technology

Maria V.

Sanchez-Vives

Universidad

Miguel

Hernandez-

CSIC, Alicante

Paul Verschure and Ulysses Bernardet ICREAUniversitat Pompeu Fabra and Barcelona Media
$\mathbf{P}$ eople who experience an immersive VR system usually report feeling as if they were really in the displayed virtual situation, and can often be observed behaving in accordance with that feeling, even though they know that they're not actually there. Researchers refer to this feeling as "presence" in virtual environments, yet the term has come to have many uses and meanings, all of which evolved from the notion of telepresence in teleoperator systems. Currently, many applications, such those that use VR in psychotherapy, rely on presence for their success. Many empirical studies have attempted to determine the impact of various technological factors-such as field-of-view, frame rate, stereo, and head tracking-on how much presence VR participants reportedly experience. Typically, researchers assess this using questionnaires. However, because there's no scientific theory that explains how or why presence occurs, application builders have no scientifically grounded guidelines on which they can build presence-inducing virtual environments.

In Presenccia, we take an operational approach to the presence concept. Our approach lets us assess the extent of presence using tools beyond traditional questionnaires, and therefore we avoid many of the problems involved with sole reliance on these. Instead, we consider the extent to which mixed reality (MR) and VR participants realistically respond to virtually generated sensory data. Specifically, we measure the similarity of their response with what we might observe or predict if the sensory data - the situation, place, or events - were real, rather than virtual. We consider this response on several levels, including the following:

brain activity, such as that measurable by functional magnetic resonance imaging;

- nonconscious physiological responses, such as heart rate and skin conductance;

- involuntary behaviors, such as flinching in response to a nearby flying object;

- voluntary behaviors, such as deciding to move closer to something of interest;

- emotional and psychological responses; and

- thoughts and higher-level cognitive responses. ${ }^{1}$

The Presenccia project's main goal is to scientifically understand presence in MR and VR environments and to use this knowledge to enhance the engineering of presence experiences. To illustrate our project's vision, we offer an extended example of a woman, Alex, traveling to a marathon by train (see the sidebar, "Presence: An Example Scenario").

\section{The Presenccia project}

Presenccia is a four-year Integrated Project of 15 European partners funded by the European Union's Sixth Framework program, Future and Emerging Technologies. FET funds long-term, 15-year research projects that can show specific useful progress within five years.

\section{Research groups}

Presenccia's 15 partners are clustered into multiple groupings with different specialties. One group of computer scientists specializes in VR-related computer graphics, haptics, and distributed systems, with expertise in presence. Partners include Departament de Llenguatges i Sistemes Informátics, Universitat Politècnica de Catalunya, Spain (UPC); Department of Computer Science, University College London (UCLCS); and the PERCRO group at Scuola di Studi Universitari e di Perfezionamento Sant 'Anna, Italy.

Another group of computer scientists concentrates on wide-area tracking and fusion of sensor data. Partners here include Computer Laboratory, University of Cambridge; Technische Universität München, Fachbereich für Informatik, Fachgebiet für Augmented Reality, Germany; and Institute for Computer Graphics and Vision, Graz University of Technology.

The neuroscientists within the project focus on two areas. Some researchers concentrate on studies recording human brain activity, including partners at the Department of Neuropsychology, University of Zurich, Switzerland (UNIZH); Karolinska Institutet, Sweden (KI); and The Human-Virtual Reality Laboratory, Technion, Israel Institute of Technology. Other neuroscience researchers focus on various aspects of perception, from the psychophysical to the cellular level in the brain. Partners here include Instituto de Neurociencias de Alicante, Universidad Miguel Hernhandez, Spain (UMH); Institute of Ophthalmology, University College London; and Chalmers University of Technology, Sweden.

There is also a group specializing in brain-computer interfaces, which includes both biomedical engineers and neuroscientists. This group researches two complementary BCI aspects. The first uses BCI to let people 
interactively effect changes in their environment through thought alone. Partners in this project are the Laboratory of Brain-Computer Interfaces, Graz University of Technology, Austria, and Guger Technologies, Austria (g.tec). The second aspect is led by researchers at Goldsmiths College, University of London, who are helping people achieve altered states (such as enhanced creativity) by feeding back aspects of brain activity into the environment (as the example story illustrates).

Another project partner, Universitat Pompeu Fabra's Institute of Audiovisual Studies, Technology Department, includes both neuroscientists and computer scientists and is taking a cognitive neuroscience approach to building an intelligent entity that is itself a large-scale MR environment that will eventually be mounted as a permanent exhibition in Barcelona. Presenccia features strong interaction among various clusters and individual institutions, with significant joint research and engineering development.

\section{Research highlights}

Given space limitations, we describe here only a few highlights from among Presenccia's many initiatives. Our focus is on emphasizing the unique and strong connections between VR researchers and neuroscientists. A lengthier version of this article, with more project examples, is available online (http://www.presenccia. org/IEEECGA).

Presence and virtual space. Researchers can study presence in many different domains. A key domain is the participant's response to the virtual space itself, in particular as it relates to spatial navigation and wayfinding. Do people move through a virtual space as if it were real? This has been an important area of research within the VR literature; VR has also been used as a tool by neuroscientists in the study of wayfinding, in particular in brain imaging studies. In Presenccia, UNIZH is studying this with humans using brain imaging, while UMH - as we now describe-is studying the topic at the cellular level.

Spatial navigation and cognition result from the integration of different sources of information in different brain areas. Neurons in the hippocampal complex that code for location are called place cells. UMH is exploring aspects of the information the brain integrates to create cognitive maps of space. With that objective, $\mathrm{UMH}$ is studying spatial processing in rats, while manipulating their environment by altering its size, shape, illumination, or meaning.

You might assume that studying navigation at the cellular level—with rodents, no less - can't be of much help in understanding what happens with humans in VR. Nevertheless, an interesting finding here immediately relates to the fact that, within VR, people typically have trouble finding their way around, and the spatial information they learn within a VE doesn't transfer well to the real world. We can infer this result from cellular-level findings that might have a neuronal correlation in the absence of the brain's theta rhythms when navigation occurs while body movement is restrained. Researchers have associated theta rhythm in the hippocampus with

\section{Presence: An Example Scenario}

When Alex arrives at the train station, she approaches the ticket office, which is staffed by virtual people who engage in friendly, efficient, and intelligent conversation. Alex finds herself smiling back at them and wishing them a good day, even though she knows that doing so makes no rational sense.

While on the train to the marathon, Alex puts on a lightweight $\mathrm{VR}$, brain-computer interface (VR-BCI) device with a marathon as the background scenario. She looks down at her virtual body, which she feels to be her own, and notices that she's wearing the wrong clothes. She visualizes her running clothes and they immediately appear on her virtual body. She joins the race and has all the feelings associated with real running, including physical tiredness. She waves to friends who are also running, and they wave back.

Alex feels her performance isn't as good as it should be; the crowds lining the route look bored, the sky is gray, and it begins to rain. She forgets about her straining muscles and burning feet and relaxes into the mental state her trainer advised her to adopt. As she does this, the sky brightens, the crowd becomes enthusiastic, and she feels in excellent physical form. Although largely mental, Alex knows that this training experience will also map over into useful physical changes that will help her in the real marathon. Her complete engagement in the virtual marathon race is interrupted when someone in the virtual crowd shouts that her train is arriving at the station. Alex takes off the equipment; as she does so, she sees a nearby train guard removing his $\mathrm{VR}-\mathrm{BCl}$ equipment as well.

sensory motor integration and as a link between egocentric and allocentric navigation. Therefore, the absence of theta rhythm might prevent the adequate generation of a cognitive map.

This finding is significant given that, in most applications, participants navigate within VEs using a joystick while physically standing or sitting still. Indeed, researchers have reported on experiments in which presence was significantly lower for a group using point-andclick to move through a VE compared with two other groups who used actual body movement associated with walking. ${ }^{2}$ We conclude that by understanding the neurophysiology underlying the creation of cognitive maps of space, we might be able to learn the basis for a virtual environment to generate spatial presence.

Projecting the body. Returning to our marathoner example, after entering the VR, Alex looked down, saw her virtual body, and felt it to be hers. How is this possible? Although we generally assume that our sense of what is and is not part of our body is quite unassailable, recent research has shown this isn't the case. We can demonstrate this with a simple experiment: Seat a volunteer at a table, hide his or her right arm behind a screen, and place a rubber arm on the table where the real arm might plausibly rest. Then, synchronously tap the back of the rubber hand and the person's real hand. After a short time, the person will start to experience the rubber hand as his or her own, ${ }^{3}$ and might even show signs of pain if the rubber hand is attacked.

This phenomenon is known as the "rubber arm illusion." Presenccia aims to apply this multisensory corre- 

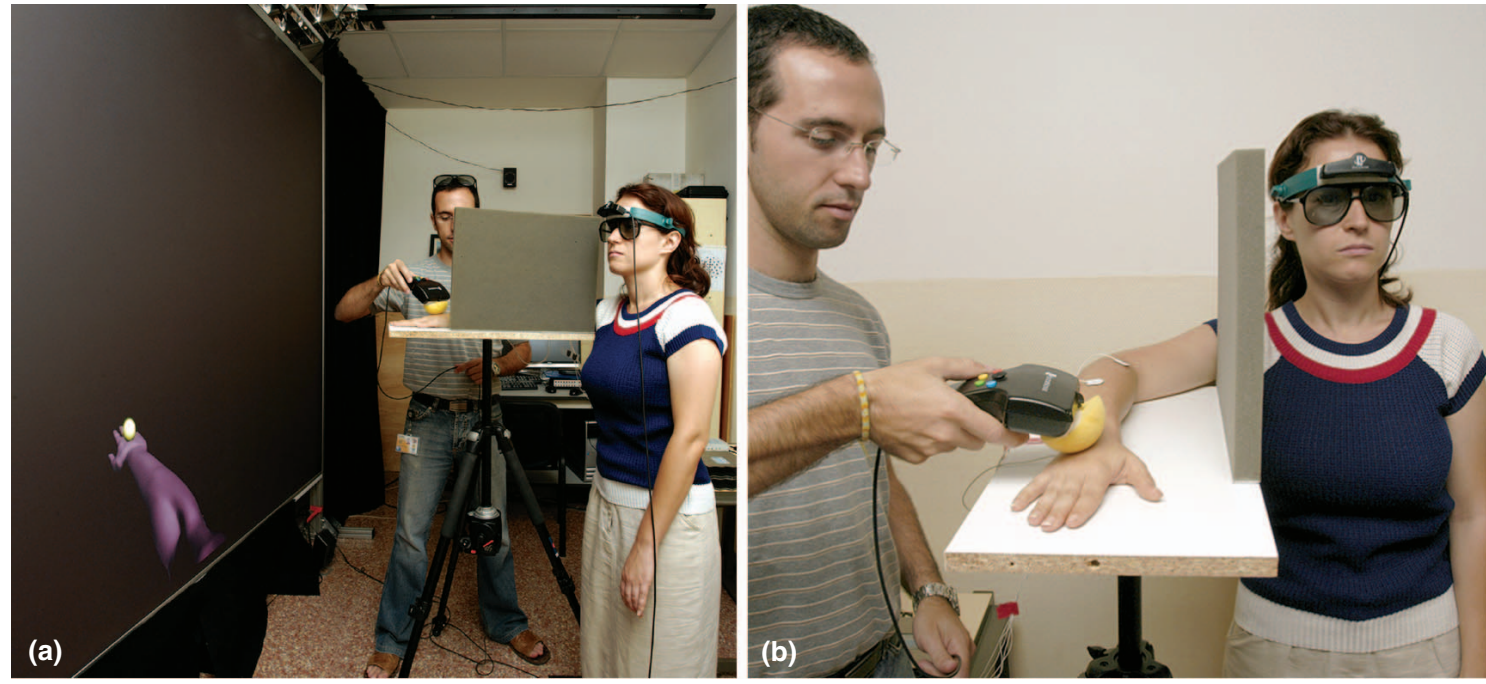

1 Recreating the "rubber arm illusion" experiment. (a) The participant sees a 3D stereo projection of an arm emerging from her right shoulder, with the room otherwise in darkness, while (b) her real arm is hidden. The experimenter uses a tracked wand to stimulate the participant's real hand while, in synchrony, a virtual ball slaved to the wand strikes the virtual hand. After a few minutes of this, most people feel the virtual arm to be their real arm-a phenomenon demonstrated in both subjective reporting and behavioral and physiological measures.

lation principle to a full virtual body. Work by UMH, UPC, and KI has already demonstrated that it's possible to do this using an arm portrayed in a projection-based VR system (see Figure 1). KI is concentrating on understanding the illusion's underlying neural mechanisms, so that, ultimately, we can apply this knowledge in an engineering solution that will deliver the illusion through VR and MR.

Brain-computer interfaces. In our marathoner example, Alex attached a BCI, thought about the clothes she wanted to wear, and they promptly appeared. This is a (rather advanced) example of a BCI in action. In

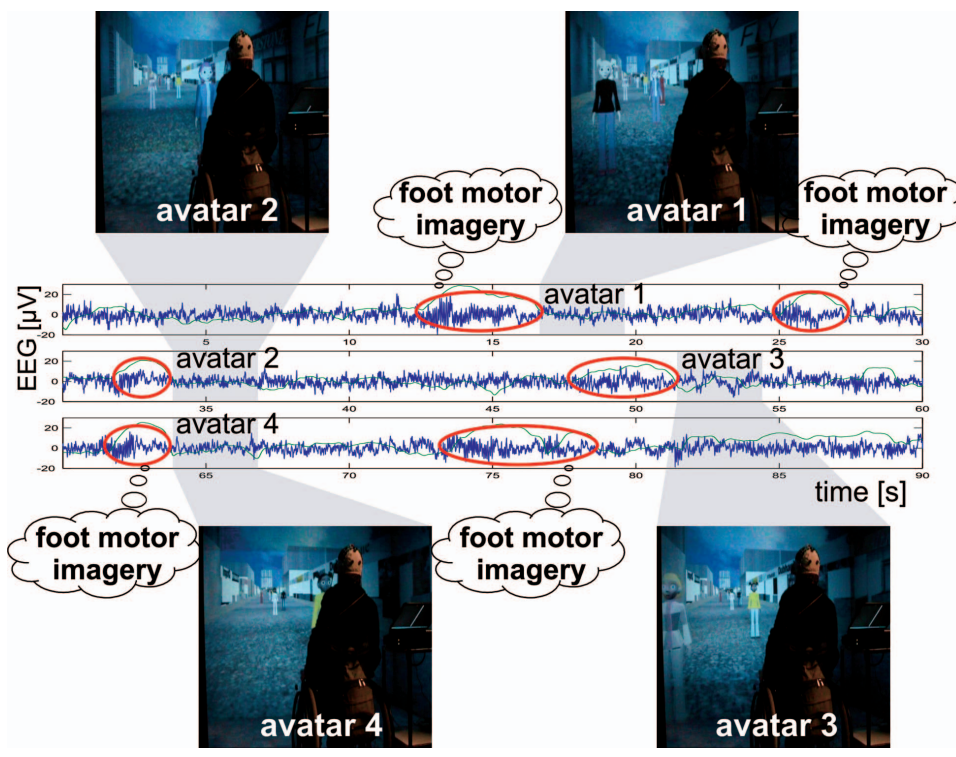

2 A wheelchair-bound person uses thought to walk through a virtual street. The EEG traces show the beta wave activation during the imagination of foot movement, which the application converted into forward movement. The participant's task was to go from one avatar to the next and have a short conversation with each one. today's practice, the fundamental idea is that an application can record EEG or other brain signals while participants are thinking specific thoughts (for example, when they're thinking about moving their hands or feet). A computer classification program learns how particular brain signal patterns are associated with particular thoughts. Then, during the operational phase, when the program recognizes a certain pattern-such as that associated with the mental imagery of foot movement-it can effect a corresponding action within either the real world (such as move a wheelchair forward) or (more safely) within a virtual world.

This method can be synchronous (the participant is cued to have an appropriate thought about hand or foot movement in response to a signal). Or, with much greater difficulty, it can be asynchronous (the participant can have the thought at any time). TUG and g.tec are carrying out this work in collaboration with UCL-CS and UPC. They've had considerable success: g.tec has developed new BCI equipment, including portable devices. They've successfully achieved the target result within $\mathrm{VR}^{4}$ and have demonstrated — for the first timethat a tetraplegic can use brain waves to control his wheelchair's movements in VR (see Figure 2).

Persistent virtual community. Our marathon scenario offers several examples of the possibilities for shared experiences and community in VR. In the virtual run, Alex recognized her running colleagues, waved to them, and they waved back. The train conductor temporarily joined the virtual world to deliver a warning relevant to Alex's real world. In addition, crowd members watching the virtual marathon rehearsal were mostly virtual characters, but also included avatars representing real online people distributed throughout the world.

Within Presenccia, we are working toward this vision by creating a persistent virtual community. This PVC is 
grounded in a particular physical installation in Barcelona; people can visit and enjoy it physically, or experience it virtually. We'll populate the PVC with virtual characters and representations of real online people. Both population types can interact with physically present humans in a specially constructed, humanaccessible interactive space.

Moreover, the PVC's overall system will itself be an intelligent entity that learns from the human participants' behavior and sometimes guides them to particular behaviors. The physical, human-accessible gateway to Presenccia's PVC extends a previous interactive space, Ada, ${ }^{5}$ into a novel infrastructure that supports the PVC in MR (see Figure 3).

Presence research has a very long way to go; researchers have yet to even adopt a common definition as to what presence is supposed to mean. This fact motivated us to adopt a particular operational definition of presence to use within the Presenccia project. Doing so gives us a concrete and empirical mechanism to assess the degree of presence engendered for an individual within any particular application. Our goal is to understand presence at every level—from the brain scan to the questionnaire.

We plan to use the knowledge gained in our scientific research to engineer application forms that maximize the chance of presence occurring. In the last analysis, if immersive VR does not deliver presence, then what's its point? If MR participants don't take the virtual objects and events seriously enough to react realistically, then those objects and events have no function (except in the limited case of pure information providers). We believe that presence is a unifying concept on the VR-MR possibilities spectrum and that the way we've defined it provides a solid foundation for scientific understanding and engineering advances in this area.

Finally, however much we concentrate on the day-today scientific activities, we should never forget VR's amazing power to transform our lives. As an example, we return to our story: Alex removes the head-mounted display and BCI and gets off the train, only to find that there's nothing beyond the platform-no city, no park, nothing. Alex experiences a profound break in presence, remembering that, in fact, she is still wearing a head-mounted display and BCI, and is still at home. Indeed, Alexander isn't a runner at all, but is actually a retired engineer trying out some new "gizmo" that his grandchildren bought him for his 89th birthday.

\section{Acknowledgments}

The European Sixth Framework Program, Future and Emerging Technologies, contract 27731, funds the Integrated Project Presenccia project. Presenccia's additional principal investigators and institutions are Henrik Ehrsson, Karolinska Institute, Sweden; John Gruzelier, Psychology Department, Goldsmiths CollegeUniversity of London; Lutz Jäncke, Department of Neuropsychology, University of Zurich, Switzerland; Mendel Kleiner, Applied Acoustics, Chalmers University of Technology, Sweden; Gudrun Klinker, Technische Universität München, Fachbereich für Informatik, Fachgebiet für Augmented Reality, Germany; Peter Robinson, Computer Laboratory, University of

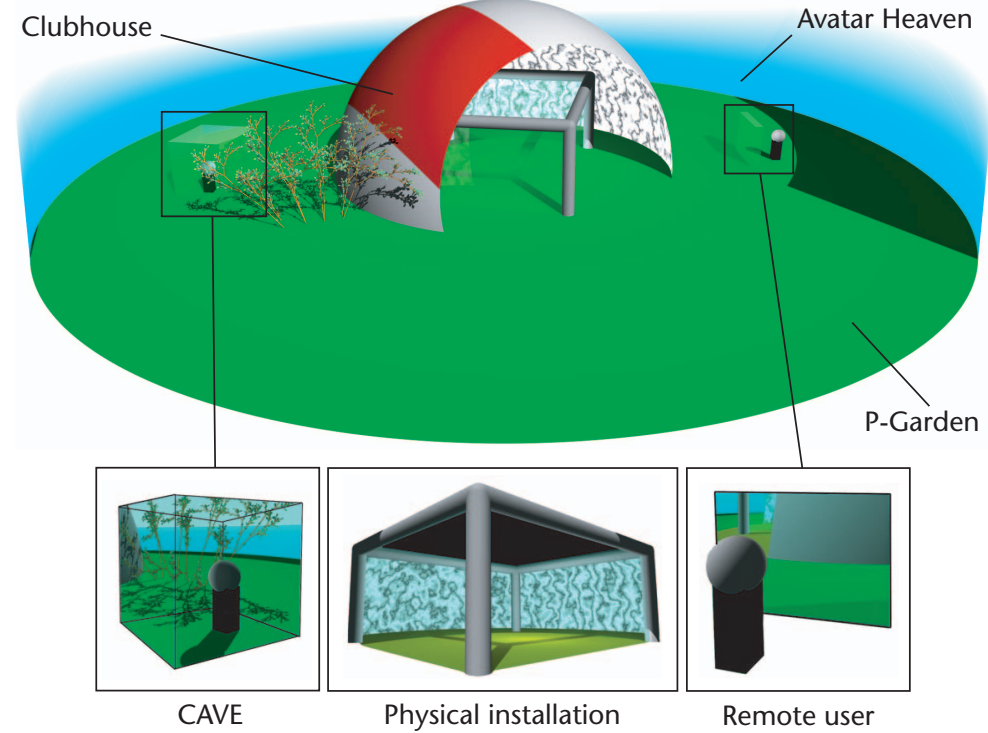

3 Conceptual overview of Prensenccia's persistent virtual community (PVC). The upper section of the figure shows the PVC's logical layout. The PVC's mixed reality world consists of the Clubhouse, the Garden, and the Avatar Heaven. The virtual counterpart of the physical installation XIM (experience induction machine) is located inside the Clubhouse. The lower section depicts the three PVC access modes: Users can either access through the physical installation, a Cave Automatic Virtual Environment (CAVE), or via the Internet from a PC (remote user). The PVC will provide a venue where entities of different degrees of virtuality - from real installation visitors to avatars and remote visitors' alter egos-can meet and interact. PVC will control fully synthetic characters using neurobiologically grounded models of perception and behavior. Ultimately, the mixed-reality installation will be open to the general public and will showcase the Presenccia project's key technologies.

Cambridge, UK; and Dieter Schmalstieg, Institute for Computer Graphics and Vision, Graz University of Technology.

\section{References}

1. M.V.Sanchez-Vives and M. Slater, "From Presence to Consciousness through Virtual Reality," Nature Reviews Neuroscience, vol. 6, no. 4, 2005, pp. 332-339.

2. M. Usoh et al., "Walking > Walking-in-Place > Flying, in Virtual Environments," Proc. 26th Int'l Conf. Computer Graphics and Interactive Techniques (Siggraph), ACM Press, 1999, pp. 359-364.

3. M. Botvinick and J. Cohen, "Rubber Hands 'Feel' Touch that Eyes See," Nature, 1998, vol. 391, no. 6669, p. 756.

4. R. Leeb et al., "Walking by Thinking: The Brainwaves are Crucial, Not the Muscles!" Presence-Teleoperators and Virtual Environments, vol. 15, no. 5, 2006, pp. 500-514.

5. K. Eng et al., "Design for a Brain Revisited: The Neuromorphic Design and Functionality of the Interactive Space 'Ada,"' Rev. Neurosci., vol. 14, nos. 1-2, 2003, pp. 145-180.

Contact author Mel Slater at melslater@gmail.com.

Contact the department editors atcga-vr@computer. org. 